\title{
Training for skeletal muscle capillarization: a Janus-faced role of exercise intensity?
}

\author{
Lasse Gliemann ${ }^{1}$ (D)
}

Received: 13 June 2016 / Accepted: 16 June 2016 / Published online: 24 June 2016

(C) Springer-Verlag Berlin Heidelberg 2016

Muscle capillarization is central for the delivery of oxygen and nutrients to the exercising muscle, and thus, capillarization is vital for exercise capacity. A high muscle capillary density means a large muscle-to-blood exchange surface area, short oxygen diffusion distance, and high red blood cell mean transit time. After all, this is what you need for translating a high cardiac output to a great exercise performance. Exercise is the most potent stimulator of angiogenesis in skeletal muscles, and few weeks of exercise training lead to measureable increases in muscle capillarization. Untrained subjects may enjoy 10-30 \% increase in capillarization after 6-8 weeks of exercise training, whereas elite aerobic athletes with years of progressive exercise training express a capillary-to-muscle fiber ratio of more than $200 \%$ of that of untrained individuals (Ingjer 1979). During exercise, vascular endothelial growth factor (VEGF) is secreted from the muscle fibres to the muscle interstitium. VEGF is the single most important growth factor for expansion of the capillary bed and together with a number of other angioregulatory factors; VEGF levels determine the degree of capillary growth (Egginton 2009).

As with all health- and performance-related interventions, researchers are keen to find the single most potent,

Communicated by Klaas R. Westerterp and Håkan Westerblad.

Based on: "Exercise duration-matched interval and continuous sprint cycling induce similar increases in AMPK phosphorylation, PGC- $1 \alpha$, and VEGF mRNA expression in trained individuals" by Taylor et al. 2016

Lasse Gliemann

Gliemann@nexs.ku.dk

1 Section for Integrative Physiology, Department of Nutrition, Exercise and Sports, University of Copenhagen, Universitetsparken 13, 2100 Copenhagen $\varnothing$, Denmark easy to do, and time-efficient method for increasing VEGF and improving muscle capillarization.

In this issue of European Journal of Applied Physiol$o g y$, Taylor et al. show that the angiogenic potential of one $120 \mathrm{~s}$ bout of continuous all-out cycling is similar to the angiogenic potential of "classic" $4 \times 30$ s all-out cycling. This is despite the fact that the $4 \times 30 \mathrm{~s}$ causes more than $50 \%$ more work being done, and a high peak power output is achieved-not just one, but four times. In this setup, skeletal muscle VEGF mRNA is increased to the same extent after the two different training protocols. Moreover, peroxisome proliferator-activated $\gamma$-receptor co-activator (PGC-1 $\alpha$ ), an important regulator of both angiogenesis and mitochondrial biogenesis, was also increased to the same extent in the two training protocols. These findings are interesting, because activation of PGC- $1 \alpha$ and subsequent mitochondrial biogenesis is largely intensity dependent (Oliveira et al. 2014), whereas angiogenesis appears to be hampered when intensity gets too high (Høier et al. 2013). As such, recent reports have shown that increasing the number of all-out efforts while reducing total training volume has a negative impact on skeletal muscle VEGF levels and ultimately capillarization (Gliemann et al. 2015). Specifically, it has been shown that the angiogenic potency of an interval-based training session is less than that of a continuous moderate-intensity training session (Høier et al. 2013). This has been shown over the past few years in various sports, including running, cycling, and soccer, and in both untrained, moderately trained, and elite athletes.

Is there a Janus-faced role of exercise intensity? Training for high cardiac output and high mitochondrial density calls for all-out efforts, whereas training for capillary growth calls for hours and hours of moderate-intensity exercise. Recall that the transport of loads of oxygen from a high cardiac output to the site of energy production is via 
the capillary bed. Thus, there appears to be a Janus-faced role of exercise intensity, where short high-intensity exercise favours cardiac remodelling and mitochondrial biogenesis, whereas longer sessions of moderate-intensity exercise favour capillarization. The mechanism(s) behind this controversy remains to be elucidated, but there are a number of likely candidates to explore. First, angiogenesis is largely dependent on the frictional force of blood applied to the luminal side of the blood vessel, termed shear stress (Egginton 2009). Shear stress is obviously very high during all-out exercise, but the duration is short. As shear stressinduced angiogenesis is a product of both duration and magnitude of the applied shear stress, hours on the bike ergometer likely outweigh the shear stress of $120 \mathrm{~s}$ all-out cycling. Second, as intensity goes up, so does the release of anti-angiogenic factors. These are released to keep capillary growth from going haywire, and it has been shown that at least some of these are upregulated more after high intensity compared to moderate-intensity exercise (Høier et al. 2013). Finally, or maybe ultimately, the aerobic component of $4 \times 30$ s all-out exercise is rather limited compared to the energy turnover from anaerobic processes (Beneke et al. 2002). Since a high capillary density is largely connected to a high aerobic energy turnover, the stimuli for capillary growth of anaerobic all-out efforts are naturally limited.

Nevertheless, high-intensity exercise holds an angiogenic potential. Although we still need to see how this potential compares to the angiogenic potential of a longer moderate-intensity exercise bout, these findings by Taylor et al. highlight that one single bout of all-out exercise is sufficient to introduce upregulation of various factors that are important for a fruitful training response. Moreover, this study indicates that the nature of an all-out protocol (continuous vs. intermittent) might not be that important for angiogenesis, let alone mitochondrial biogenesis.

In the light of Taylor et al., variation and periodisation of training make even more sense. Whether you are an aerobic athlete with hours in the saddle, a soccer player with numerous short energy bursts, or an untrained couch potato, high capillarization aids the delivery of oxygen, removal of waste products, and may even fight insulin resistance.

\section{References}

Beneke R, Pollmann C, Bleif I et al (2002) How anaerobic is the Wingate anaerobic test for humans? Eur J Appl Physiol 87:388-392. doi:10.1007/s00421-002-0622-4

Egginton S (2009) Invited review: activity-induced angiogenesis. Pflug Arch Eur J Physiol 457:963-977. doi:10.1007/ s00424-008-0563-9

Gliemann L, Gunnarsson TP, Hellsten Y, Bangsbo J (2015) 10-20 30 training increases performance and lowers blood pressure and VEGF in runners. Scand J Med Sci Sports 25:e479-e489. doi: $10.1111 / \mathrm{sms} .12356$

Høier B, Passos M, Bangsbo J, Hellsten Y (2013) Intense intermittent exercise provides weak stimulus for vascular endothelial growth factor secretion and capillary growth in skeletal muscle. Exp Physiol 98:585-597. doi:10.1113/expphysiol.2012.067967

Ingjer F (1979) Capillary supply and mitochondrial content of different skeletal muscle fiber types in untrained and endurancetrained men. A histochemical and ultrastructural study. Eur J Appl Physiol Occup Physiol 40:197-209

Oliveira NRC, Marques SO, Luciano TF et al (2014) Treadmill training increases SIRT-1 and PGC-1 $\alpha$ protein levels and AMPK phosphorylation in quadriceps of middle-aged rats in an intensity-dependent manner. Mediat Inflamm 2014:987017-987111. doi:10.1155/2014/987017 